\title{
AGILITY \\ Dynamic Full Body Locomotion and Manipulation with Autonomous Legged Robots
}

\section{Conference Paper}

Author(s):

Hutter, Marco (D); Bloesch, Michael; Buchli, Jonas; Semini, Claudio; Bazeille, Stephane; Righetti, Ludovic; Bohg, Jeannette

Publication date:

2013

Permanent link:

https://doi.org/10.3929/ethz-a-009996472

Rights / license:

In Copyright - Non-Commercial Use Permitted

Originally published in:

https://doi.org/10.1109/SSRR.2013.6719336 


\title{
AGILITY - Dynamic Full Body Locomotion and Manipulation with Autonomous Legged Robots
}

\author{
Marco Hutter*, Michael Bloesch*, Jonas Buchli ${ }^{\dagger}$, \\ Claudio Semini ${ }^{\ddagger}$, Stephane Bazeille ${ }^{\ddagger}$, Ludovic Righetti ${ }^{\S}$, Jeannette Bohg ${ }^{\S}$ \\ *Autonomous Systems Lab, ${ }^{\dagger}$ Agile \& Dexterous Robotics Lab, ETH Zürich, Switzerland, mahutter@ethz.ch \\ ${ }_{\ddagger}^{\ddagger}$ Department of Advanced Robotics, Istituto Italiano di Tecnologia (IIT), Italy

 \\ All authors contributed equally to this work
}

\begin{abstract}
This paper presents the vision of the recently launched project AGILITY that aims to join forces across Europe to bring robots with legs and arms into highly unstructured outdoor environment such as a disaster area. Building upon state of the art torque controllable quadrupedal robots, we jointly investigate environment perception, motion planning, and whole body control strategies that enable rough terrain locomotion and manipulation. The developed machines will be able to autonomously navigate through challenging ground by optimally adapting the contact forces to the ground (e.g., propping feet against an obstacle), by using whole body motions to extend their standard workspace (e.g., twisting the body to reach), or by dynamic maneuvers (e.g., jumping or leaping). The proposed methods will be evaluated in a rescue scenario using (future versions of) our torque controllable quadrupedal robots $\mathrm{HyQ}$ and StarlETH.
\end{abstract}

\section{INTRODUCTION}

Recent natural and human made disasters (e.g., Fukushima 2011) have tragically shown that the current robot technology provides only minimal support when it comes to operation in unstructured and challenging disaster-like environment. It turns out that, despite the extensive technological tools and machines we have at hand, simple navigation in unknown and rough terrain is barely achievable with available solutions.

Motivated by these deficiencies, different large scale projects have been initiated to support research and robotics development for such scenarios. The obvious and most advanced solution is to use (small scale) flying robots to quickly assess the situation [1, 2] in a disaster area. This has the advantage that the robots are not in contact with the environment and can hence traverse nearly arbitrary terrain. However, flying vehicles need large air space (in comparison to their body size) which prevents them from working in very cluttered environment. Furthermore, small flying robots have very limited payload capabilities, which makes it impossible to carry large sensor equipment or to manipulate the environment. Moreover, recent experiments with flying and crawling robots in a church of the earthquake-hit Mirandola (Italy, NIFTi European project [3]) have shown that aerial vehicles can send a lot of dust into

This research is partially supported by the Swiss National Science Foundation through the National Centre of Competence in Research Robotics and an SNF professorship Award to J.B., ETH Zürich, the Fondazione Istituto Italiano di Tecnologia and the Max Planck Society. We gratefully acknowledge the contributions of C. David Remy to early versions and the vision of this project. the air that limits visibility. These problems can be overcome by using ground vehicles. The currently most elaborated ones use tracks and flippers to cope with highly unstructured terrain [3, 4, 5]. While having large advantages due to a rather simple maneuverability, such systems lack in mobility when it comes to ground that requires climbing or discrete contact point selection. As a solution for this locomotion challenge, the robotics community attributes great potential to legged robots for ground operation in unstructured and challenging terrain. There are quite a number of past and ongoing projects in the field of rescue robotics. One of the better known and funded projects is the recently launched DARPA Robotics Challenge (DRC) [6], which supports different teams with humanoid robots that are supposed to work semi-autonomously in a postdisaster environment.

In AGILITY, we target a similar scenario but use quadrupedal robots, which we endow with (simple) manipulation capabilities. We focus on fundamental solutions to research questions that arise with the underlying dynamic full body locomotion and manipulation.

\section{Project Outline}

Providing successful robotic solutions for a search and rescue scenario requires to combine progress in:

- hardware development of robust and versatile platforms that are suited for operation in rough environment,

- behavior planning and control to quickly and safely navigate the machines across challenging terrain,

- manipulation and grasping to act on the environment and to support the body for special maneuvers,

- state estimation, visual tracking, and mapping to precisely localize and map the robot and its environment.

In AGILITY we jointly address these engineering and research challenges with experts in all domains. We deploy autonomous quadrupedal robots equipped with sophisticated sensors and manipulators. These force controllable multilegged systems have the large advantage that they can interact with the environment through multiple and continuously changing contact points. Like humans that tend to use their hands when it comes to traversing challenging ground, the additional contact with the environment allows to augment the locomotion capabilities. It improves stability, enlarges the 


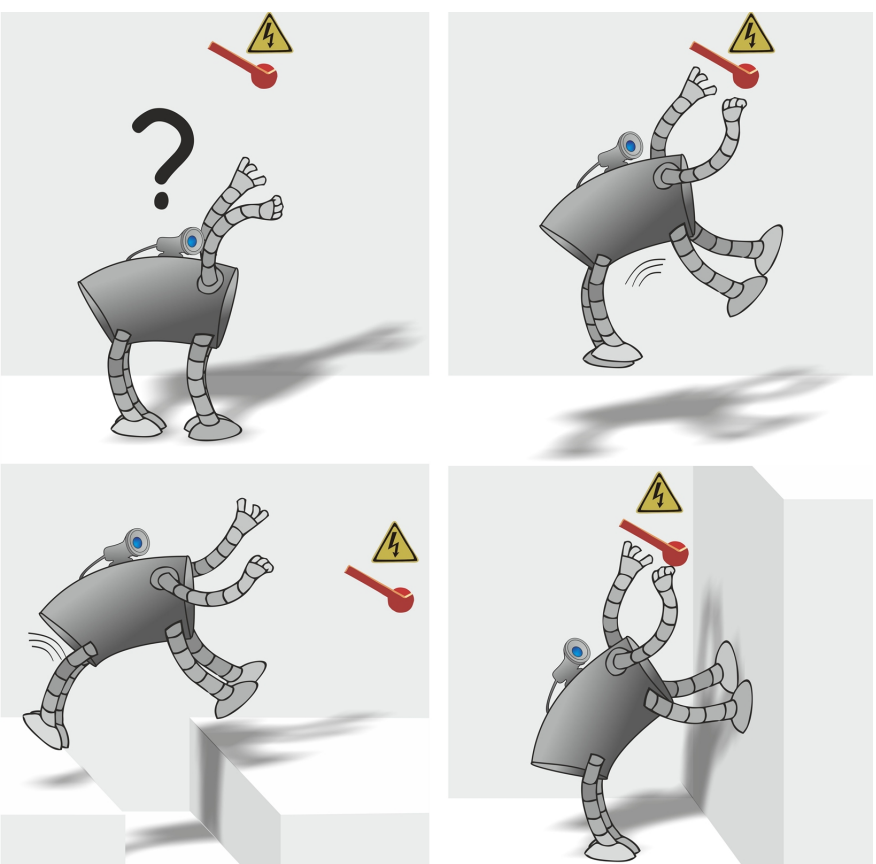

Fig. 1. Being able to perform dynamic full body manipulation on legged robots will greatly improve the versatility and reach of today's systems.

range of operation, and provides a solid stance if required for (heavy-duty) manipulation.

Using novel whole body control techniques, the powerful and torque controllable legged platforms are supposed to traverse rough terrain by optimally exploiting the multi-contact situation (e.g., propping feet against an obstacle), to extend their workspace (e.g., twisting the body to reach), and to perform dynamic maneuvers (e.g., jumping or leaping). During locomotion, simultaneously they can use their arms and hands to actively manipulate the (unknown) environment such as opening a door or operating a switch (Fig. 11. Locomotion and manipulation will be tackled as a combined problem using floating base whole body kinematics and dynamics. This allows for simultaneous and coordinated execution of locomotion and manipulation tasks which enables a performance that cannot be achieved with wheeled or tracked vehicles.

Search and rescue robots have to deal with a highly unstructured and a priori unknown environment. To enable autonomous locomotion, the quadrupedal robots combine sensor information from stereo cameras, laser sensors, IMU, joint encoders, and, very importantly, joint torque and contact force sensors to get precise estimation about the robot's state as well as a map of its environment. For manipulation, we envision to enhance the precision by actively tracking objects and endeffectors as well as to increase the versatility by reactive control algorithms based on contact force measurements.

\section{PROJECT PARTNERS}

The AGILITY project has been launched in July 2013 as a research collaboration between four research labs at ETH Zurich, IIT Genova, and MPI Tubingen. These four labs bring together a unique body of knowledge and expertise relevant to the presented goals.

\section{A. ETH-ADRL}

The Agile \& Dexterous Robotics Labs (ETH-ADRL) research activities focus on understanding the control of dexterous, dynamic and precise motions of robots and humans in unstructured environments. This understanding provides the "intelligence" for future versatile, agile and dexterous service robots. ADRL has extensive expertise on whole-body modelbased control of walking robots [7, 8, 9], impedance, force and torque control on multi-DOF robots [10, 11], applied machine learning [10, 12, 13, 14] and adaptive control applied to locomotion [15], manipulation [16] and modeling of human motor control [17]. ETH-ADRL will furthermore provide its expertise on low-level torque control and software development as well as machine learning techniques.

\section{B. ETH-ASL}

The Autonomous Systems Lab (ETH-ASL) is internationally renowned in the field of design, control, and navigation of autonomous robots operating in different types of environments. The ASL has a strong expertise in (visual) localization and mapping for rough terrain navigation [e.g. 18] that recently culminated in a SLAM sensor for precise localization an mapping in a disaster like environment [19]. In addition to this, ASL performs research on the design [20] and wholebody control [e.g. 21, 22] of torque controllable legged robots.

\section{IIT-ADVR}

The Department of Advanced Robotics (IIT-ADVR) is active in a wide range of robotic research with a focus on legged robots. It has excellent skills in the design of articulated robots such as iCub, COMAN/C-cub robot or the hydraulically actuated quadruped robot HyQ [23]. The engineering and research expertise at ADVR includes light-weight mechanical construction, force/torque sensor and electronics development, as well as hydraulic actuation. Furthermore, the department has extensive knowledge in high-bandwidth torque control with hydraulic and electric actuators [24, 25], floating-base rigid body dynamics [26], impedance control [27], design of real-time control software for complex robotic systems [28] and experience in indoor and outdoor experiments of various walking, jumping and running robots [e.g. 26, 29, 30].

\section{MPI-IS AMD}

The Autonomous Motion Department at the Max-PlanckInstitute for Intelligent Systems (MPI-IS AMD) is pursuing research on the principles of perception, action and learning in autonomous systems that successfully interact with complex environments. It has substantial expertise in machine learning applied to robotic manipulation and grasping [31, 32] as well as to legged locomotion [9]. Furthermore, the research expertise includes nonlinear torque control of complex robots [33] and vision-based robotic grasp and manipulation planning [34, 35, 36, 37]. The department's experience in real-time tactile and visual sensing has lead to the development of methods towards robust closed-loop grasp execution such as online movement adaptation [38] and visual servoing [39]. 


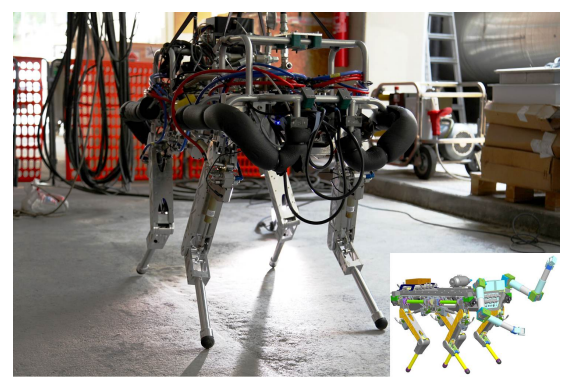

(a) HyQ (IIT-ADVR) [23]

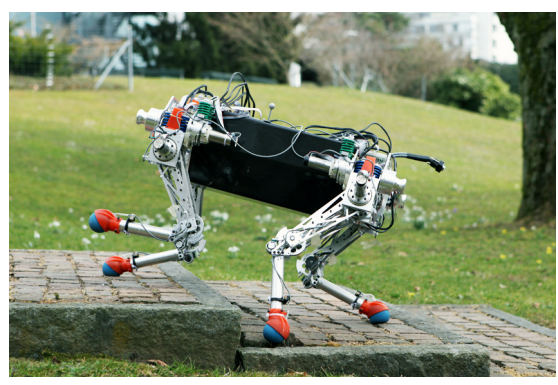

(b) StarETH (ETH-ASL) [20]

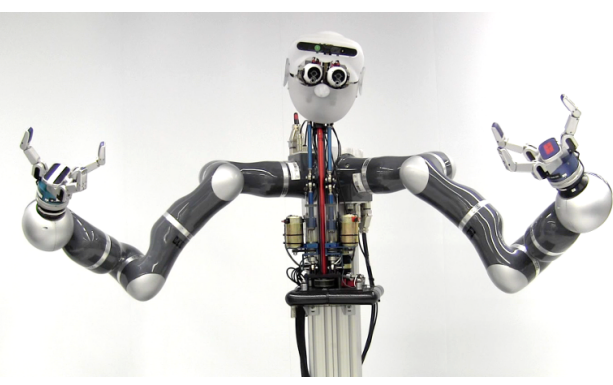

(c) Apollo (MPI)

Fig. 2. The consortium's torque-controllable robotic platforms: (a) HyQ - quadruped based on hydraulic actuation, (b) StarlETH - quadruped based on series elastic actuation, (c) Apollo - dual-arm manipulation platform with two Kuka LBR IV, two three-fingered Barrett Hands and a Sarcos Humanoid head.

\section{Preliminary Results}

The AGILITY consortium can build upon several ground breaking results in legged robot design, whole-body control, manipulation, and visual-perception.

Over the past five years, IIT-ADVR and ETH-ASL have developed the state of the art hydraulically and electrically actuated quadrupedal robots HyQ [23](Fig. 2(a)] and StarlETH [20](Fig. 2(b)). This new generation of precisely torque controllable, robust, and compliant legged systems is ideally suited for interaction with rough and unknown terrain. Both systems are capable of different gaits such as static walking or fast and dynamic running [e.g. 21, 22, 26]. Furthermore, due to powerful joint actuation, they are suited to perform highly dynamic maneuvers such as jumping on or off an obstacle. They have sufficient payload capabilities for long autonomy and to carry sophisticated sensors. Within AGILITY, both quadrupedal robots will be equipped with a custom made sensor head that includes stereo cameras, laser sensor, and IMU. HyQ will be additionally extended with two hydraulic arms that allow to actively manipulate the environment as required to open doors or to move stones out of the way.

To control the robots, the AGILITY consortium can count on expert skills in whole-body control of legged platforms. MPI-IS has published several ground-breaking research results on inverse dynamics techniques that allow to generate sophisticated behaviors with complex robotic systems [33]. ETH-ASL presented (hierarchical) optimization strategies that were applied on StarlETH to achieve dynamic locomotion [21, 22] as well as to statically walk in rough terrain while optimizing the contact force distribution [40]. ETH-ADRL has demonstrated how to use inverse dynamics techniques to generate a compliant behavior that enhances the climbing capabilities as well as robustness against unperceived obstacles [7]. IIT-ADVR has presented a reactive locomotion controller framework based on inverse dynamics techniques that resulted in very stable rough terrain trotting and balancing with HyQ both on a treadmill and outdoors [26].

In terms of manipulation, MPI-IS has shown how machine learning can enable compliant and reliable grasping maneuvers [31, 36] and how vision-based tracking of objects as well as the endeffector position can increase precision [39, 41].
Furthermore, MPI-IS studied how different sensor modalities can be exploited or fused for increasing the robustness of grasping [37, 38, 42]. This research has been evaluated on different manipulation platforms. At MPI-IS, the dual-arm robot Apollo as depicted in Fig. 2(c) is available for conducting further research on manipulation and grasping in dynamic and cluttered environments.

All outlined control strategies rely on precise state estimation about where the robot is and how the local terrain looks like. By fusing kinematic information with IMU measurements (acceleration and rotation rate), we could show that the robot can reliably estimate its current state [43] and also precisely detect slippage at the ground contact points [44]. Additionally, the combination of visual and IMU measurements [18, 19] allows to robustly determine the egomotion of the robot and to continuously build up a point cloud of the environment. In AGILITY, these two features are combined.

On the perception side, mechanical image stabilization with an active head and preliminary results on terrain mapping with stereo cameras have been obtained. Furthermore, two visionenhanced locomotion behavior on HyQ have recently been published [30, 45].

\section{CONCLUSION}

The goal of AGILITY is to foster research on dynamic full body locomotion and manipulation with autonomous quadrupedal robots by joining forces across Europe in a targeted research collaboration between four groups at ETH, MPI, and IIT. While the project focuses on fundamental research questions, the consortium aims at developing for and applying these techniques to search and rescue scenarios. AGILITY serves as a catalyst for advanced legged platform design, whole body control techniques, manipulation and grasping in unknown and cluttered environments, as well as state estimation, mapping, and visual tracking. In contrast to challenge oriented programs (e.g. DRC), our efforts aim at solving fundamental research questions and providing a foundation for a wide spread application of legged mobile manipulation for service robotics at large. This ranges from a common hard- and software framework to the same control concepts that are (partially already) used by all project partners. 


\section{REFERENCES}

[1] SFly, "sFly - small and safe helicopters to assist rescue and monitoring."

[2] Sherpa, "Sherpa - mixed ground and aerial robotic platforms to support search and rescue."

[3] NIFTi, "NIFTi - Natural Human-Robot Cooperation in Dynamic Environments."

[4] K. Nagatani, A. Yamasaki, K. Yoshida, T. Yoshida, and E. Koyanagi, "Semi-autonomous traversal on uneven terrain for a tracked vehicle using autonomous control of active flippers," in International Conference on Intelligent Robots and Systems, pp. 2667-2672, Sept. 2008.

[5] B. M. Yamauchi, "PackBot: a versatile platform for military robotics," in SPIE (G. R. Gerhart, C. M. Shoemaker, and D. W. Gage, eds.), pp. 228237, Sept. 2004

[6] DRC, "DARPA Robotics Challenge (DRC)."

[7] J. Buchli, M. Kalakrishnan, M. Mistry, P. Pastor, and S. Schaal, "Compliant quadruped locomotion over rough terrain," in Int. Conference on Intelligent Robots and Systems (IROS), pp. 814-820, 2009.

[8] M. Mistry, J. Buchli, and S. Schaal, "Inverse dynamics control of floating base systems," IEEE Int. Conference on Robotics and Automation (ICRA), pp. 3406-3412, 2010.

[9] M. Kalakrishnan, J. Buchli, P. Pastor, M. Mistry, and S. Schaal, "Learning, planning, and control for quadruped locomotion over challenging terrain," International Journal of Robotics Research, vol. 30, pp. 236258, 2011.

[10] J. Buchli, S. Stulp, E. Theodorou, and S. Schaal, "Learning variable impedance control," Int. J. Robotics Research, vol. 30, no. 7, pp. 820 833, 2011

[11] L. Righetti, J. Buchli, M. Mistry, and S. Schaal, "Inverse dynamics control of floating-base robots with external constraints: a unified view," in Proc. of the IEEE Int. Conference on Robotics and Automation (ICRA), pp. 1085-1090, 2011.

[12] M. Kalakrishnan, J. Buchli, and S. Schaal, "Learning locomotion on rough terrain using terrain templates," in International Conference on Intelligent Robots and Systems (IROS), pp. 167-172, 2009.

[13] E. Theodorou, J. Buchli, and S. Schaal, "a generalized path integral control approach to reinforcement learning," Journal of Machine Learning Research, no. 11, pp. 3137-3181, 2010.

[14] F. Stulp, J. Buchli, E. Theodorou, and S. Schaal, "Reinforcement learning of full-body humanoid motor skills," International Conference on Humanoid Robots (Humanoids), 2010.

[15] J. Buchli and A. Ijspeert, "Self-organized adaptive legged locomotion in a compliant quadruped robot," Autonomous Robots, vol. 25, no. 4, pp. 331-347, 2008.

[16] F. Stulp, E. Theodorou, J. Buchli, and S. Schaal., "Learning to grasp under uncertainty," IEEE Int. Conference on Robotics and Automation (ICRA)., 2011

[17] F. Stulp, J. Buchli, A. Ellmer, M. Mistry, E. Theodorou, and S. Schaal, "Reinforcement learning of impedance control in stochastic force fields," in Development and Learning (ICDL), 2011 IEEE International Conference on, vol. 2, pp. $1-6$, aug. 2011.

[18] R. Voigt, J. Nikolic, C. Hurzeler, S. Weiss, L. Kneip, and R. Siegwart, "Robust embedded egomotion estimation," in IEEE/RSJ Int. Conference on Intelligent Robots and Systems (IROS), pp. 2694-2699, Sept. 2011.

[19] J. Nikolic, M. Burri, J. Rehder, S. Leutenegger, C. Hürzeler, and R. Siegwart, "UAV System for Inspection of Industrial Facilities," in IEEE Aerospace, 2013.

[20] M. Hutter, C. Gehring, M. Bloesch, M. H. Hoepflinger, C. D. Remy, and R. Siegwart, "StarlETH: a Compliant Quadrupedal Robot for Fast, Efficient, and Versatile Locomotion," in International Conference on Climbing and Walking Robots (CLAWAR), pp. 483-490, 2012.

[21] M. Hutter, C. Gehring, M. H. Hoepflinger, M. Bloesch, and R. Siegwart, "Walking and Running with StarlETH," in International Symposium on Adaptive Motion of Animals and Machines (AMAM), 2013.

[22] C. Gehring, S. Coros, M. Hutter, M. Bloesch, M. H. Hoepflinger, and R. Siegwart, "Control of Dynamic Gaits for a Quadrupedal Robot," in IEEE International Conference on Robotics and Automation (ICRA), (Karlsruhe, Germany), 2013.

[23] C. Semini, N. G. Tsagarakis, E. Guglielmino, M. Focchi, F. Cannella, and D. G. Caldwell, "Design of HyQ - a hydraulically and electrically actuated quadruped robot," IMechE Part I: Journal of Systems and Control Engineering, vol. 225, no. 6, pp. 831-849, 2011.

[24] T. Boaventura, C. Semini, J. Buchli, M. Frigerio, M. Focchi, and D. G Caldwell, "Dynamic torque control of a hydraulic quadruped robot," in Int. Conference on Robotics and Automation (ICRA), 2012.
[25] M. Focchi, T. Boaventura, C. Semini, M. Frigerio, J. Buchli, and D. G. Caldwell, "Torque-control based compliant actuation of a quadruped robot," in IEEE International Workshop on Advanced Motion Control $(A M C), 2012$

[26] V. Barasuol, J. Buchli, C. Semini, M. Frigerio, E. R. De Pieri, and D. G. Caldwell, "A reactive controller framework for quadrupedal locomotion on challenging terrain," in IEEE Int. Conf. on Robotics and Automation (ICRA), 2013.

[27] T. Boaventura, G. A. Medrano-Cerda, C. Semini, J. Buchli, and D. G. Caldwell, "Stability and performance of the compliance controller of the quadruped robot hyq," in IEEE/RSJ International Conference on Intelligent Robots and Systems (IROS), 2013. (accepted).

[28] M. Frigerio, J. Buchli, and D. G. Caldwell, "Code generation of algebraic quantities for robot controllers," in IEEE/RSJ International Conference on Intelligent Robots and Systems (IROS), 2012.

[29] C. Semini, H. Khan, M. Frigerio, T. Boaventura, M. Focchi, J. Buchli, and D. G. Caldwell, "Design and scaling of versatile quadruped robots," in Int. Conf. on Climbing and Walking Robots (CLAWAR), 2012.

[30] S. Bazeille, V. Barasuol, M. Focchi, I. Havoutis, M. Frigerio, J. Buchli, C. Semini, and D. Caldwell, "Vision enhanced reactive locomotion control for trotting on rough terrain," in IEEE International Conference on Technologies for Practical Robot Applications (TePRA), 2013.

[31] M. Kalakrishnan, L. Righetti, P. Pastor, and S. Schaal, "Learning Force Control Policies for Compliant Manipulation," in Proc. of the IEEE/RSJ Int. Conference on Intelligent Robots and Systems (IROS), pp. 46394644, 2011

[32] J. Bohg and D. Kragic, "Learning grasping points with shape context," Robotics and Autonomous Systems, vol. 58, no. 4, pp. 362 - 377, 2010

[33] L. Righetti, J. Buchli, M. Mistry, M. Kalakrishnan, and S. Schaal, "Optimal distribution of contact forces with inverse dynamics control," International Journal of Robotics Research (IJRR), 2013.

[34] J. Bohg, M. Johnson-Roberson, B. León, J. Felip, X. Gratal, N. Bergström, D. Kragic, and A. Morales, "Mind the Gap - Robotic Grasping under Incomplete Observation," in Proc. of the IEEE Int. Conference on Robotics and Automation (ICRA), 2011.

[35] J. Bohg, K. Welke, B. León, M. Do, D. Song, W. Wohlkinger, M. Madry, A. Aldóma, M. Przybylski, T. Asfour, H. Martí, D. Kragic, A. Morales, and M. Vincze, "Task-based grasp adaptation on a humanoid robot," in Int. IFAC Symposium on Robot Control (SYROCO), Sep 2012.

[36] A. Herzog, P. Pastor, M. Kalakrishnan, L. Righetti, T. Asfour, and S. Schaal, "Template-Based Learning of Grasp Selection," in IEEE Int. Conf. on Robotics and Automation (ICRA), 2012.

[37] L. Righetti, M. Kalakrishnan, P. Pastor, J. Binney, J. Kelly, R. C. Voorhies, G. Sukhatme, and S. Schaal, "An Autonomous Manipulation System based on Force Control and Optimization," 2013. In submission.

[38] P. Pastor, L. Righetti, M. Kalakrishnan, and S. Schaal, "Online Movement Adaptation based on Previous Sensor Experiences," in Int. Conference on Intelligent Robots and Systems (IROS), pp. 365 - 371, 2011.

[39] X. Gratal, J. Romero, J. Bohg, and D. Kragic, "Visual servoing on unknown objects," IFAC Mechatronics: The Science of Intelligent Machines, 2011.

[40] M. Hutter, M. H. Hoepflinger, C. D. Remy, and R. Siegwart, "Hybrid Operational Space Control for Compliant Legged Systems," in RSS Robotics Science and Systems, (Sydney, Australia), 2012.

[41] M. Wühtrich, P. Pastor, M. Kalakrishnan, J. Bohg, and S. Schaal, "Probabilistic object tracking using a range camera," in Proc. of the IEEE/RSJ Int. Conference on Intelligent Robots and Systems (IROS), 2013. accepted.

[42] J. Ilonen, J. Bohg, and V. Kyrki, "3-d object reconstruction of symmetric objects by fusing visual and tactile sensing," in IEEE International Conference on Robotics and Automation (ICRA'13), May 2013.

[43] M. Bloesch, M. Hutter, M. H. Hoepflinger, S. Leutenegger, C. Gehring, C. D. Remy, and R. Siegwart, "State Estimation for Legged Robots Consistent Fusion of Leg Kinematics and IMU," in Robotics Science and Systems (RSS), (Sydney, Australia), 2012.

[44] M. Bloesch, C. Gehring, P. Fankhauser, M. Hutter, M. Hoepflinger, and R. Siegwart, "Robust State Estimation for Legged Robots," in IEEE/RSJ Intenational Conference on Intelligent Robots and Systems (IROS), 2013.

[45] I. Havoutis, J. Ortiz, S. Bazeille, V. Barasuol, C. Semini, and D. G. Caldwell, "Onboard perception-based trotting and crawling with the hydraulic quadruped robot (hyq)," in IEEE/RSJ International Conference on Intelligent Robots and Systems (IROS), 2013. 